\title{
Detection of Minimal Residual Disease in the
}

\section{Peripheral Blood of Breast Cancer Patients, with a Multi Marker (MGB-I, MGB-2, CK-19 and NY-BR-I) Assay}

\author{
Suzy E Meijer ${ }^{1,2}$ \\ Olga Klebanov-Akopyn ${ }^{3}$ \\ Vera Pavlov ${ }^{2,3}$ \\ Shachar Laks ${ }^{4}$ \\ David Hazzan ${ }^{4}$ \\ Aviram Nissan ${ }^{2-4}$ \\ Douglas Zippel $\mathbb{D}^{3,4}$

\begin{abstract}
'Department of Infectious Disease, Sourasky Medical Center, Tel Aviv, Israel; ${ }^{2}$ The Surgical Oncology Laboratory, Hadassah-Hebrew University Hospital, Jerusalem, Israel; ${ }^{3}$ The Surgical Oncology Laboratory, The Chaim Sheba Medical Center, Ramat Gan, Israel; ${ }^{4}$ Department of General and Oncological Surgery Surgery C, The Chaim Sheba Medical Center, Ramat Gan, Israel
\end{abstract}

Purpose: Minimal residual disease (MRD) refers to micrometastases that are undetectable by conventional means and is a potential source of disease relapse. This study aimed to detect the presence of breast cancer (BC) biomarkers (MGB-1, MGB-2, CK-19, NY-BR-1) using real-time polymerase chain reaction (RT-PCR) in peripheral blood mononuclear cells (PBMC) of BC patients and the impact of a positive assay on clinical outcome.

Patients and Methods: Patients in the analysis included females $>18$ years of age with biopsy-proven carcinoma of the breast. A $10 \mathrm{~mL}$ sample of venous blood was obtained from 10 healthy controls and 25 breast cancer patients. Comparisons of peripheral blood markers were made with clinicopathological variables.

Results: High-quality RNA was extracted from all samples with a mean RNA concentration of $224.8 \pm 155.3 \mathrm{ng} / \mu \mathrm{L}$. Each of the molecular markers examined was highly expressed in the primary breast tumors $(n=3$, positive controls) with none of the markers detected in healthy negative controls. The NY-BR-1 marker was expressed in one (4\%) patient with metastatic disease with no MGB-1 and MGB-2 detected in any sample derived from the study patients. The CK-19 marker was detected in $16(64 \%)$ of the BC cases. No correlation was found between CK-19 expression and tumor stage $(\mathrm{P}=0.07)$ or nodal status $(\mathrm{P}=0.32)$. No correlation was identified in the $\mathrm{BC}$ patients between $\mathrm{CK}-19$ expression and receptor status in the BC primary tumor.

Conclusion: This study showed high expression of all 4 markers NY-BR-1, MGB-1, MGB2 and CK-19 in the PBMCs derived from breast cancer patients. CK-19 was detected in 64\% of the stage I-III cases operated with curative intent, the only recurrent events occurring in the CK-19-positive cases. Our data confirm the need to enhance techniques for detection of MRD, which may better predict patients at risk for relapse.

Keywords: breast cancer, circulating tumor cells, cancer biomarkers, early detection, predictive potential

\section{Introduction}

\section{The Need for Enhanced Breast Cancer Detection}

Breast cancer remains a significant 21 st century global health care concern, imposing a great burden on health care systems and negatively impacting the lives of millions of patients worldwide. This has created a need for new avenues of research to help realize the goal of better disease diagnosis, therapy, and management. The 
last few decades have witnessed the early detection of breast cancer (BC) combined with significant improvements in its treatment and a consequent reduction in cancer-specific mortality. ${ }^{1}$ Despite these advances, about onequarter of non-metastatic patients go on to develop distant metastases. In clinical BC practice, the traditional predictive markers of disease recurrence and survival have included the primary tumor size, the presence of lymph node metastases and histological tumor grade along with the routine determination of receptor status. ${ }^{2}$ Improved techniques designed to detect micrometastases within lymph nodes have assisted in defining high-risk patients suitable for systemic therapy. This may help identify those cases undergoing curative surgery with negative axillary nodes, which could potentially develop distant metastases. In these patients, the detection of this minimal residual disease (MRD) disease within the lymph nodes can be achieved by serial sectioning, immunohistochemistry or by RT-PCR mRNA amplification of BC markers. ${ }^{3}$ Similarly, improved diagnostic tools utilizing such innovative techniques, such as liquid biopsies to detect potential cancer biomarkers, including cell free nucleic acids (DNA, mRNA and miRNA) in circulation, have the potential to enhance diagnosis, monitor efficacy of treatment and discover potential targets for therapy. ${ }^{4,5}$

\section{Minimal Residual Disease}

The MRD concept proposes that micrometastases undetectable by conventional means are present at the time of the initial diagnosis and that they are the source of disease relapse and a target for adjuvant therapy. ${ }^{6}$ Immunomagnetic separation of isolated tumor cells in the bone marrow (DTCs) or in the peripheral blood (CTCs) has been used as a biomarker in early-stage BC. For example, Nadal et $\mathrm{al}^{7}$ showed the presence of CTCs in nearly one-third of patients with $\mathrm{T} 1$ or $\mathrm{T} 2$ primary tumors.

Although detection of these CTCs in patients with nonmetastatic BC does not appear to correlate with tumor size, grade, with receptor status, or lymph node status, there is an inverse correlation between the extent of their presence and the relapse-free survival. ${ }^{8}$ The aim of this study was to develop a multi-marker RT-PCR assay for the detection of $\mathrm{MRD}$ in the peripheral blood of BC patients, using RNA panels to detect the presence of breast cancer biomarkers.

\section{Biomarker Panels}

MGB-1 and MGB-2 are members of the uteroglobin protein family ${ }^{9}$ that have been shown to be overexpressed in human $\mathrm{BC}$ and in $\mathrm{BC}$ cell lines ${ }^{9}$ with MGB RT-PCR correlating with histology in positive lymph nodes. ${ }^{10}$ The marker CK 19 is a non-specific epithelial marker present on every $\mathrm{BC}$ cell with its detection in the peripheral blood of $\mathrm{BC}$ patients correlating with a reduced disease-free and overall cancer-specific survival in node-negative patients. $^{11}$

NY-BR-1, first detected by Jäger et $\mathrm{al}^{12}$ in a serological analysis of recombinant tumor cDNA expression libraries (SEREX) screen showing tissue-restricted mRNA expression, was used as a novel marker particularly expressed in BC, normal testis and in prostate cancer. NY-BR-1 is considered a differentiation antigen showing expression in normal breast epithelium, in most cases of ductal carcinoma in situ (DCIS) and in many invasive breast cancer specimens. $^{13}$

\section{RT-PCR Assay for MRD}

The approach to use RT-PCR detection of multiple markers with $\mathrm{BC}$ and epithelial specificity may improve the overall sensitivity of MRD diagnosis. Previous work by our group has shown high sensitivity for a multi-marker RT-PCR assay in the detection of MRD within sentinel axillary lymph nodes. ${ }^{14}$ We present the development of a multi-marker RT-PCR assay for the detection of MRD in peripheral blood mononuclear cells (PBMC) of $\mathrm{BC}$ patients and the impact of a positive assay on clinical outcome.

\section{Patients and Methods}

The study protocol was approved by the Hadassah-Hebrew University Institutional Review Board (Helsinki Committee, protocol \#391-04-08-06), with all the study patients signing an IRB-approved informed consent. Patients in the analysis included females $>18$ years of age with biopsy-proven carcinoma of the breast. Demographic data were collected including age at diagnosis and ethnicity, along with clinicopathological features, such as anatomic locale of the tumor (laterality), preoperative CEA and CA 15-3, the number of involved lymph nodes, the number of resected lymph nodes, the pathologic stage, histologic type, grade and ER, PR and Her2/neu expression status.

A $10 \mathrm{~mL}$ sample of venous blood was obtained from 10 healthy controls without disease and preoperatively from all participating study patients. After centrifugation at $1500 \mathrm{rpm}$ for 10 minutes at room temperature, peripheral blood mononuclear cells (PBMCs) were separated on 
a Ficoll-Hypaque gradient (Amersham Biosciences AB, Uppsala Sweden) with further centrifugation at $2700 \mathrm{rpm}$ for 30 minutes. Cells were washed twice in PBS with repeat centrifugation for 7 minutes at $1500 \mathrm{rpm}$ suspending the cell pellet in $1 \mathrm{~mL}$ of TriReagent (Molecular Res Inc., Cincinnati, OH). Specimens were stored at $-20^{\circ} \mathrm{C}$ until RNA extraction. RNA was extracted with the addition of $200 \mu \mathrm{L}$ chloroform $(119.38 \mathrm{~g} / \mathrm{mL}$, Mallinckrodt Baker B.V., Deventer Holland) per $\mathrm{mL}$ of TriReagent and incubated for 15 minutes at room temperature. After centrifugation at $12,000 \mathrm{rpm}$ for 15 minutes at $4^{\circ} \mathrm{C}$ the aqueous phase was transferred to a fresh tube and mixed with $0.5 \mathrm{~mL}$ isopropanol (isopropyl alcohol-2-propanol; Frutarom Ltd., Haifa Israel) for further 8 minutes of incubation at room temperature and then repeat centrifugation at $12,000 \mathrm{rpm}$ at $4^{\circ} \mathrm{C}$ for additional 15 minutes. After a further wash in $75 \%$ ethanol, the pellet obtained was dried in a SpeedVac chamber heater and resuspended in DEPC-treated water (50-200 $\mu \mathrm{L}$ depending upon the size of the pellet). The pellet was incubated for further 15 minutes in a $65^{\circ} \mathrm{C}$ heat block and was then stored at $-70^{\circ} \mathrm{C}$ until processing.

For RNA analysis $5 \mu \mathrm{L}$ of the sample was heated for 15 minutes and then run in a $1 \%$ agarose electrophoresis gel with an $\Phi x /$ HindIII DNA marker (New England, BioLabs Inc.). The concentration and purity of the product was assessed with a NanoDrop ND 1000 spectrophotometer (NanoDrop Technologies USA) using the ND-1000 version 3.2.1. software (Coleman Technologies Inc.). Prior to cDNA synthesis, the RNA was DNase treated by incubating $1 \mu \mathrm{g}$ RNA with up to $7 \mu \mathrm{L}$ of DEPC-treated water, $1 \mu \mathrm{L}$ of DNase buffer and $1 \mu \mathrm{L}$ of amplification grade DNase (Invitrogen Carlsbad CA) for 15 minutes at room temperature. The reaction was transferred for further 15 minutes in a heat block and then stopped by the addition of $1 \mu \mathrm{L}$ of $25 \mathrm{mM}$ EDTA (Invitrogen Carlsbad, CA). For cDNA synthesis, $3 \mu \mathrm{L}$ of random primer $(\mathrm{p}(\mathrm{dN})$ 6-digoxigenin; Roche Diagnostics $\mathrm{GmbH}$, Mannheim Germany) was incubated at $70^{\circ} \mathrm{C}$ for 10 minutes after which a mix of $4 \mu \mathrm{L}$ of Strand buffer (Invitrogen Carlsbad, CA), $2 \mu \mathrm{L}$ DTT and $1 \mu \mathrm{L}$ PCR-grade dNTP (pH 8.3; Roche Diagnostics, GmbH Mannheim, Germany) were added. Following further 3 minutes of incubation at $42^{\circ} \mathrm{C}, 1 \mu \mathrm{L}$ of reverse transcriptase (TaqMan microRNA reverse transcription kit, Applied Biosystems Foster City, CA) was added with incubation for additional 50 minutes at $42^{\circ} \mathrm{C}$ (the last 15 minutes at $70^{\circ} \mathrm{C}$ ). The $20 \mu \mathrm{L}$ mix of cDNA was stored at $-20^{\circ} \mathrm{C}$ until used for RealTime PCR.
To conduct RT-PCR analysis, all primers were tested on positive and negative controls in order to determine their capacity and consistency for amplifying the sequence of interest. The primers used included NY BR-1, MGB-1, MGB-2 and CK 19 with GAPDH, the housekeeping gene used as an internal control for the normalized quantitative measurement of RNA expression. A cDNA volume equivalent to $0.1 \mu \mathrm{g}$ RNA was added to the tubes resulting in a mix containing $7 \mu \mathrm{L}$ DDW, $10 \mu \mathrm{L}$ Mastermix (TaqMan Applied Biosystems, Branchburg NJ) and $1 \mu \mathrm{L}$ of the relevant probe and primers. Optical strips or plates containing $20 \mu \mathrm{L}$ per sample were analyzed with an RTPCR thermocycler (7500 RT-PCR Applied Biosystems) with the program run in 2 stages for 40 cycles (Stage 1 $=10$ minutes at $95^{\circ} \mathrm{C}$ and Stage $2=15$ seconds at $95^{\circ} \mathrm{C}$ followed by 60 seconds at $60^{\circ} \mathrm{C}$ ). The 7500 System Software package (7500 System Sequence Detection version 1.2.2., Applied Biosystems) was used for the analysis.

Comparisons of peripheral blood markers were made with clinicopathological variables. Patients underwent routine physical examination at 3 monthly intervals for the first year after surgery and then at 6 monthly intervals for the next 4 years. Breast imaging, including mammography and ultrasonography, was performed annually postsurgery.

Statistical analysis was performed using the SPSS version 10.0 statistical package (SPSS Inc., Chicago IL). Categorical variables were compared with the Chi-square test or Fisher's Exact test where appropriate. A p value $<0.05$ was considered to be significant.

\section{Results}

The analysis included 25 patients, 24 with invasive BC and one case of DCIS (median age 57 years, range 36-71 years). Of the cohort, 20 underwent surgery and adjuvant therapy in accordance with their pathologic stage and grade. The clinicopathological features of the operated patient group are shown in Table 1. The 5 remaining cases presented with metastatic disease and were treated with chemotherapy. Over a median follow-up of 41.3 months (range 34.4-74.7 months) there were 2 distant recurrences in the operated patients, both with lung metastases.

RNA was successfully extracted from all blood samples and quantified using the housekeeping gene with a mean concentration of $224.8 \pm 155.3 \mathrm{ng} / \mu \mathrm{L}$. The sensitivity of each assay was derived from the generated calibration curve (Supplementary Figure 1). Breast cancer 
Table I Clinicopathologic Features of Study Patients Operated on for Localized Breast Cancer

\begin{tabular}{|c|c|}
\hline \multicolumn{2}{|l|}{ Laterality } \\
\hline Right & $8(40.0)$ \\
\hline Left & $10(50.0)$ \\
\hline Bilateral & $2(10.0)$ \\
\hline \multicolumn{2}{|l|}{ Hormonal status } \\
\hline Premenopausal & $8(40)$ \\
\hline Perimenopausal & $2(10)$ \\
\hline Postmenopausal & $10(50)$ \\
\hline \multicolumn{2}{|l|}{ Procedure } \\
\hline Modified radical mastectomy & $3(15)$ \\
\hline Lumpectomy & $17(85)$ \\
\hline \multicolumn{2}{|l|}{ Axillary procedure } \\
\hline SLNB only & II (55) \\
\hline ALND & $2(10)$ \\
\hline SLNB followed by ALND & $7(35)$ \\
\hline \multicolumn{2}{|l|}{ AJCC-UICC T-stage } \\
\hline Tis & I (5) \\
\hline TI & $4(20)$ \\
\hline $\mathrm{T} 2$ & $12(60)$ \\
\hline T3 & $3(15)$ \\
\hline \multicolumn{2}{|l|}{ AJCC-UICC N-stage } \\
\hline No & II (55) \\
\hline $\mathrm{NI}$ & $7(35)$ \\
\hline N2 & $2(10)$ \\
\hline \multicolumn{2}{|l|}{ Histologic grade * } \\
\hline Well differentiated & $\mathrm{I}(5.3)$ \\
\hline Moderately differentiated & $13(68.4)$ \\
\hline Poorly differentiated & $5(26.3)$ \\
\hline Undifferentiated & $0(0)$ \\
\hline \multicolumn{2}{|c|}{ Hormone receptor expression } \\
\hline ER/PR positive & $18(90)$ \\
\hline ER/PR negative & $2(10)$ \\
\hline \multicolumn{2}{|l|}{ Her2/neu status } \\
\hline Over-expressed & $4(20)$ \\
\hline No over-expression & $16(80)$ \\
\hline
\end{tabular}

Notes: *For invasive carcinomas $(n=19)$. () Brackets are percentages. Abbreviations: SLNB, sentinel lymph node biopsy; ALND, axillary lymph node dissection.

cells (MD 134) were mixed with the PBMCs derived from healthy donors $(n=10)$ in increasing concentrations with each molecular marker used capable of a threshold detection of a minimum of $10^{3}$ MDA 134 cells per $10^{6}$ PBMCs.
Table 2 Correlation Between CK-19 Expression in Sera of Operated Breast Cancer Patients and Their Primary Tumor T-Stage

\begin{tabular}{|l|c|c|c|}
\hline CK-19 Expression & T 0-I & T 2-3 & Total \\
\hline Negative & 3 & 2 & 5 \\
Positive & 2 & 13 & 15 \\
Total & 5 & 15 & 20 \\
\hline
\end{tabular}

Note: The two-sided Fisher's exact test was 0.07 .

The quantification was normalized against the PBMCs derived from healthy donors. Slight differences in the pattern and sensitivity of RNA expression between the markers were noted when cell samples were seeded with varying populations of MDA134-positive controls. NY-BR -1 amplification increased with an increase in cell numbers, but which fell away after an MDA 134 concentration exceeded $10^{5}$ cells. MGB-1 was amplified in increasing amounts until an MDA 134 concentration exceeding $10^{5}$ cells, with MGB-2 showing a higher amplification until an MDA 134 cell concentration of $5 \times 10^{5}$ cells was reached (Supplementary Figure 2).

High-quality RNA was extracted from all samples with a mean RNA concentration of $224.8 \pm 155.3 \mathrm{ng} / \mu \mathrm{L}$. Each of the molecular markers examined was highly expressed in the primary breast tumors ( $\mathrm{n}=3$, positive controls) with none of the markers detected in the PBMCs derived from healthy negative controls.

The NY-BR-1 marker was expressed in one $(4 \%)$ patient with metastatic disease with no MGB-1 and MGB-2 detected in any sample derived from the study patients. The CK-19 marker was detected in 16 (64\%) of the BC cases. No correlation was found between CK-19 expression and tumor stage $(p=0.07)$ or nodal status $(p=$ 0.32) (Tables 2 and 3). No correlation was identified in the $\mathrm{BC}$ patients between CK-19 expression and receptor status in the $\mathrm{BC}$ primary tumor. Four of the 5 metastatic patients were negative for all molecular markers with the remaining cases showing both CK-19 and NY-BR-1

Table 3 Correlation Between CK-19 Expression in Sera of Operated Breast Cancer Patients and Their Primary Tumor N-Stage

\begin{tabular}{|l|c|c|c|}
\hline CK-19 Expression & N 0 & N I-2 & Total \\
\hline Negative & 4 & I & 5 \\
Positive & 7 & 8 & 15 \\
Total & II & 9 & 20 \\
\hline
\end{tabular}

Note: The two-sided Fisher's exact test was 0.32 . 
expression. Over a median follow-up of 41.3 months (range 34.4-74.7 months) recurrence occurred in 2 cases, both presenting with lung metastases. Both of these cases were CK-19-positive, and both were managed with chemotherapy (diagnosed 13 and 37 months post-surgery).

\section{Discussion}

This pilot study showed a high expression of all 4 markers NY-BR-1, MGB-1, MGB-2 and CK-19 in the PBMCs derived from 25 women with invasive $\mathrm{BC}$ and DCIS. CK-19 was detected in $64 \%$ of the Stage I-III cases operated with curative intent and was the only marker consistently identified. Two metastatic recurrent events occurred in patients, both of whom were CK-19-positive.

\section{Relevance of the Study}

The detection of CTCs in the peripheral blood of patients with early-stage $\mathrm{BC}$ is prognostic for recurrence and cancer-specific mortality ${ }^{8}$ with the potential for use in monitoring response and resistance to individual anticancer therapies. ${ }^{7,15}$ The findings in this study show differences for MRD site in $\mathrm{BC}$ where our group has previously reported the use of a multi-marker assay in sentinel lymph nodes incorporating NY-BR-1, MGB-2 and CK19. ${ }^{14}$ In this earlier study, 15 of 30 cases demonstrated all 3 markers with a higher sensitivity for MRD detection within lymph nodes when compared with either conventional histology or immunohistochemistry. In this study, NY-BR-1 was the most sensitive marker with CK-19 the least sensitive and with an advantage for a 3-marker over a single-marker detection system.

\section{Comparative Findings}

Our results are in keeping with those reported by Krüger et $\mathrm{al}^{16}$ who detected CK-19 mRNA transcripts in the peripheral blood of $46 \%$ of $\mathrm{BC}$ cases. These findings are also in agreement with those reported by Stathopoulou et $\mathrm{al}^{17}$ who identified CK-19 transcripts in one-third of their operable BC patients. A fall in specificity between laboratories will account for variations in the reported false-positive rates. ${ }^{18,19}$ The assumption that the dissemination of CK-19-positive CTCs is an early event, which signifies the risk of relapse, requires further evaluation in order to determine the potential benefit in early $\mathrm{BC}$ patients of quantitative CTC-marker RT-PCR as a method for monitoring adjuvant therapy. This approach adopted by Xenidis et $\mathrm{al}^{11,20}$ has suggested an association between detectable post-chemotherapy CK-19 mRNA- positive CTCs and axillary lymph node status, as well as with disease-free and overall survival.

Zach et $\mathrm{al}^{21}$ were the first to establish a nested PCR assay designed to detect hMAM mRNA in the peripheral blood of BC patients, demonstrating a correlation between the presence of positive transcripts and tumor burden. In contrast, we were unable to show any mammaglobin (MGB) CTC expression. Our results are consistent with those reported by Chen et $\mathrm{al}^{22}$ who were unable to correlate the presence of MGB-positive CTCs with the outcome. However, unlike our findings, these researchers showed MGB positivity in $26 \%$ of their early BC cases. Similarly, Marques et al, ${ }^{23}$ taking samples before the commencement of adjuvant chemotherapy, were unable to show any difference in hMAM CTC-positivity between those later developing recurrence and patients who were disease-free. This is in contrast to Ntoulia et $\mathrm{al}^{24}$ who showed an inverse correlation in operable BC patients between the expression of Mammaglobin-A in the peripheral blood and the disease-free interval. This variability likely reflects the conflicting associations that have been demonstrated between the expression of hMAM in the peripheral blood and more typical $\mathrm{BC}$ prognostic markers. In a study by Nuñez-Villar et $\mathrm{al}^{25}$ hMAM expression correlated with indicators of a good prognosis (ER and PR receptor expression, diploid DNA content, a low Ki67 labeling and low tumor grade), whereas Mikhitarian et al showed hMAM overexpression to be associated with ER receptor negativity and a higher tumor grade. ${ }^{26}$

The multi-marker assay approach has generally shown more consistent results than the use of a single marker assay. In a study of early BC patients using a similar approach to ours, but with a combination of 3 established mRNA markers (CK-19, human mammaglobin-hMAM and carcinoembryonic antigen-CEA) Chen et $\mathrm{al}^{22}$ showed a $58.8 \%$ sensitivity and a $100 \%$ specificity for CTC detection in the peripheral blood with a clear correlation between initial multi-marker positivity and distant relapse over a 3-year follow-up. In their study, the detection of 3-marker-positive CTCs in the peripheral blood was an independent risk factor, which correlated with a reduced relapse-free survival. Multi-marker assay detection systems are likely to have a significant advantage over single marker assays for several reasons. For the detection of MDR, multi-marker RT-PCR should show enhanced performance characteristics, particularly when cell populations are phenotypically diverse and when the concentrations of expressed mRNA are low. The use of 
a number of markers can potentially compensate for these variations and allow CTC detection to act as a better marker for the molecular characterization of systemic disease. $^{27}$

Even when some markers have been reported in combination as independent prognostic factors for survival, their sensitivity can be too low for clinical use as single markers. Differences encountered reflect different patient subpopulations, the detection of CTCs with techniques that have variable performance and particular situations where no information is available concerning the comparative level of marker expression in the primary tumor. In general, multi-marker RT-PCR analysis of the peripheral blood shows high specificity and is associated with histologic grade, lymph node metastasis and higher levels of serum tumor markers, such as CEA and CA 15-3. ${ }^{28}$ Furthermore, positive peripheral blood markers used in combination can predict progression-free survival ${ }^{29}$ and in women with Stages I-III BC a positive multi-marker assay prior to the commencement of adjuvant chemotherapy correlates with a poor disease-free survival. ${ }^{30}$

There are several limitations to our study. Patient numbers are small, and the data are preliminary and need confirmation in larger cohorts. In addition, there are clear technical considerations, which influence test sensitivity. A major challenge in detecting MRD is that a standard blood sample can only identify a few thousand copies of each gene. It is accepted, however, that cancer cells have a poorer survival than normal cells, potentially interfering with PCR amplification. Additional strategies designed for epithelial enrichment will also enhance CTC marker expression and improve test sensitivity and subsequent clinical utility. ${ }^{31-33}$ Other technical considerations such as the timing of processing of samples, the sequence of blood tube collection, prolonged sample storage prior to assay and the type of blood preservative can all affect results, particularly when the positivity rate for an individual marker is low. ${ }^{34,35}$

Our data add to the ever-increasing usage of a range of varied "omic" technologies such as genomics, transcriptomics, proteomics and metabolomics, to more precisely characterize tumor biology. ${ }^{36}$ Indeed, due to the complexity of human biology, new advances in interpreting these data, and integrating and combining different methodologies, a process known as multi-omics has become an essential innovation in cancer research. Recently, this approach has been used to develop a molecular signature to differentiate between young women at high or low risk for breast cancer development, which similarly could pave the way for better early detection. ${ }^{37}$

\section{Conclusion}

In summary, our study showed CK-19 marker expression in peripheral blood CTCs in early-stage BC. The only recurrent events occur in the CK-19-positive cases. Further validation is required to establish an approach where the molecular detection of CTCs and genomic monitoring of their clearance during treatment will act as surrogate markers for systemic disease. In this way, tracking the appearance of CTCs can expand the use of targeted agents to patients who, according to standard criteria, would normally be ineligible for such treatment. ${ }^{38}$ RTPCR marker analysis in early-stage BC will potentially permit personalized cancer management and the monitoring of resistance to anticancer therapies. Large clinical trials using RT-PCR CTC detection as a sensitive endpoint will need to establish adjuvant therapies directed by genomic characterization result in significant improvements in clinical outcome.

\section{Ethics Statement}

All work on this manuscript was done in accordance with the Declaration of Helsinki.

\section{Disclosure}

The authors report no conflicts of interest in this work.

\section{References}

1. Winters S, Martin C, Murphy D, et al. Breast cancer epidemiology, prevention, and screening. Prog Mol Biol Transl Sci. 2017;151:1-32.

2. Vuong D, Simpson PT, Green B, et al. Molecular classification of breast cancer. Virchows Arch. 2014;465(1):1-14. doi:10.1007/s00428014-1593-7

3. Parsons HA, Rhoades J, Reed SC, et al. Sensitive detection of minimal residual disease in patients treated for early-stage breast cancer. Clin Cancer Res. 2020;26:2556-2564. doi:10.1158/1078-0432.CCR-193005

4. Schwarzenbach H, Hoon D, Pantel K. Cell-free nucleic acids as biomarkers in cancer patients. Nat Rev Cancer. 2011;11(6):426-437. doi: $10.1038 / \mathrm{nrc} 3066$

5. Crigna AT, Samec M, Koklesova L, et al. Cell-free nucleic acid patterns in disease prediction and monitoring-hype or hope? EPMA J. 2020;11(4):1-25.

6. Ignatiadis M, Reinholz M. Minimal residual disease and circulating tumor cells in breast cancer. Breast Cancer Res. 2011;13:222. doi:10.1186/bcr2906

7. Nadal R, Lorente JA, Rosell R, et al. Relevance of molecular characterization of circulating tumor cells in breast cancer in the era of targeted therapies. Expert Rev Mol Diagn. 2013;13:295-307. doi:10.1586/erm.13.7 
8. Hall CS, Karhade MG, Bowman Bauldry JB, et al. Prognostic value of circulating tumor cells identified before surgical resection in nonmetastatic breast cancer patients. J Am Coll Surg. 2016;223:20-29. doi:10.1016/j.jamcollsurg.2016.02.021

9. Hassan EM, Willmore WG, McKay BC, et al. In vitro selections of mammaglobin A and mammaglobin B aptamers for the recognition of circulating breast tumor cells. Sci Rep. 2017;7:14487. doi:10.1038/ s41598-017-13751-z

10. Daniele L, Annaratone L, Allia E, et al. Technical limits of comparison of step-sectioning, immunohistochemistry and RT-PCR on breast cancer sentinel nodes: a study on methacarn-fixed tissue. J Cell Mol Med. 2009;13:4042-4050. doi:10.1111/j.1582-4934.2008.00449.x

11. Xenidis N, Perraki M, Kafousi M, et al. Predictive and prognostic value of peripheral blood cytokeratin-19 mRNA-positive cells detected by real-time polymerase chain reaction in node-negative breast cancer Patients. J Clin Oncol. 2006;24:3756-3762. doi:10.1200/JCO.2005.04.5948

12. Jäger D, Stockert E, Gure AO, et al. Identification of a tissue-specific putative transcription factor in breast tissue by serological screening of a breast cancer library. Cancer Res. 2001;61:2055-2061.

13. Jäger D, Filonenko V, Gouth I, et al. NY-BR-1 is a differentiation antigen of the mammary gland. Appl Immunohistochem Mol Morphol. 2007;15:77-83. doi:10.1097/01.pai.0000213111.05108.a0

14. Nissan A, Jäger D, Roystacher M, et al. Multimarker RT-PCR assay for the detection of minimal residual disease in sentinel lymph nodes of breast cancer patients. $B r \quad J$ Cancer. 2006;94:681-685. doi:10.1038/sj.bjc.6602992

15. Banys-Paluchowski M, Krawczyk N, Fehm T. Potential role of circulating tumor cell detection and monitoring in breast cancer: a review of the current evidence. Front Oncol. 2016;6:255. doi: $10.3389 /$ fonc. 2016.00255

16. Krüger W, Krzizanowski C, Holweg M, et al. Reverse transcriptase/ polymerase chain reaction detection of cytokeratin-19 mRNA in bone marrow and blood of breast cancer patients. J Cancer Res Clin Oncol. 1996;122:679-686. doi:10.1007/BF01209032

17. Stathopolou A, Mavroudis D, Perraki M, et al. Molecular detection of cancer cells in the peripheral blood of patients with breast cancer: comparison of CK-19, CEA and maspin as detection markers. Anticancer Res. 2003;23:1883-1890.

18. Schoenfeld A, Kruger KH, Gomm J, et al. The detection of micrometastases in the peripheral blood and bone marrow of patients with breast cancer using immunohistochemistry and reverse transcriptase chain reaction for keratin 19. Eur J Cancer. 1997;33:854-861. doi:10.1016/S0959-8049(97)00014-2

19. Krüger WH, Jung R, Detlefsen B, et al. Interference of cytokeratin-20 and mammaglobin-reverse-transcriptase polymerase chain assays designed for the detection of disseminated cancer cells. Med Oncol. 2001;18:33-38. doi:10.1385/MO:18:1:33

20. Xenidis M, Ignatiadis M, Apostolaki S, et al. Cytokeratin-19 mRNA positive circulating tumor cells after adjuvant chemotherapy in patients with early breast cancer. J Clin Oncol. 2009;27:2177-2184. doi:10.1200/JCO.2008.18.0497

21. Zach O, Kasparu H, Krieger O, et al. Detection of circulating mammary carcinoma cells in the peripheral blood of breast cancer patients via a nested reverse transcriptase polymerase chain reaction assay for mammaglobin mRNA. J Clin Oncol. 1999;17:2015-2019. doi:10.1200/JCO.1999.17.7.2015

22. Chen Y, Zou TN, Wu ZP, et al. Detection of cytokeratin 19, human mammaglobin and carcinoembryonic antigen-positive circulating tumor cells by three-marker reverse transcription-PCR assay and its relation to clinical outcome in early breast cancer. Int J Biol Markers. 2010;25:59-68. doi:10.1177/172460081002500201
23. Marques AR, Teixeira E, Diamond J, et al. Detection of human mammaglobin mRNA in serial peripheral blood samples from patients with non-metastatic breast cancer is not predictive of disease recurrence. Breast Cancer Res Treat. 2009;114:223-232. doi:10.1007/s10549-008-0002-9

24. Ntoulia M, Stathopoulou A, Ignatiadis M, et al. Detection of Mammaglobin A-mRNA-positive circulating tumor cells in peripheral blood of patients with operable breast cancer with nested RT-PCR. Clin Biochem. 2006;39:879-887. doi:10.1016/j. clinbiochem.2006.06.009

25. Núñez-Villar MJ, Martínez-Arribas F, Pollán M, et al. Elevated mammaglobin (hMAM) expression in breast cancer is associated with clinical and biological features defining a less aggressive tumour phenotype. Breast Cancer Res. 2003;5:R65-70. doi:10.1186/bcr587

26. Mikhitarian K, Martin RH, Ruppel MB, et al. Detection of mammaglobin mRNA in peripheral blood is associated with high grade breast cancer: interim results of a prospective cohort study. BMC Cancer. 2008;8:55. doi:10.1186/1471-2407-8-55

27. Liang DH, Hall C, Lucci A. Circulating tumor cells in breast cancer. Rec Results Cancer Res. 2020;215:127-145.

28. Zhao S, Yang H, Zhang M, et al. Circulating tumor cells (CTCs) detected by triple-marker EpCAM, CK 19 and hMAM RT-PCR and their relation to clinical outcome in metastatic breast cancer patients. Cell Biochem Biophys. 2013;65:263-273. doi:10.1007/s12013-0129426-2

29. Zhao S, Liu YP, Zhang QY, et al. The prognostic role of circulating tumor cells (CTCs) detected by RT-PCR in breast cancer: a meta-analysis of published literature. Breast Cancer Res Treat. 2011;130:809-816. doi:10.1007/s10549-011-1379-4

30. Ignatiadis M, Kallergi G, Ntoulia M, et al. Prognostic value of the molecular detection of circulating tumour cells using a multimarker reverse transcription-PCR assay for Cytokeratin 19, Mammaglobin A, and HER2 in early breast cancer. Clin Cancer Res. 2008;19:2593-2600. doi:10.1158/1078-0432.CCR-07-4758

31. Melo JV, Yan XH, Diamond J, et al. Reverse transcription/polymerase chain reaction (RT/PCR) amplification of very small numbers of transcripts: the risk in misinterpreting negative results. Leukemia. 1996;10:1217-1221.

32. Reinholz MM, Nibbe A, Jonart LM, et al. Evaluation of a panel of tumor markers for molecular detection of circulating cancer cells in women with suspected breast cancer. Clin Cancer Res. 2005;11:3722-3732. doi:10.1158/1078-0432.CCR-04-1483

33. Hall C, Valad L, Lucci A. Circulating tumor cells in breast cancer patients. Crit Rev Oncog. 2016;21:125-139. doi:10.1615/ CritRevOncog.2016016120

34. Benoy IH, Elst H, Van Dam P, et al. Detection of circulating tumour cells in blood by quantitative real-time RT-PCR: effect of pre-analytical time. Clin Chem Lab Med. 2006;44:1082-1087. doi:10.1515/CCLM.2006.210

35. Li GL, Zhang J, Jin KT, et al. Human mammaglobin: a superior marker for reverse transcriptase PCR in detecting tumor cells in breast cancer patients. Biomark Med. 2011;5:249-260. doi:10.2217/bmm.11.20

36. Karczewski KJ, Snyder MP. Integrative omics for health and disease. Nat Rev Genet. 2018;19:299-310. doi:10.1038/nrg.2018.4

37. Fröhlich H, Patjoshi S, Yeghiazaryan K, et al. Premenopausal breast cancer: potential clinical utility of a multi-omics based machine learning approach for patient stratification. EPMA J. 2018;11:175-186.

38. Bozionellou V, Mavroudis D, Perraki M, et al. Trastuzumab administration can effectively target chemotherapy-resistant cytokeratin-19 messenger RNA-positive tumor cells in the peripheral blood and bone marrow of patients with breast cancer. Clin Cancer Res. 2004;10:8185-8194. doi:10.1158/1078-0432.CCR-03-0094 


\section{Publish your work in this journal}

Breast Cancer - Targets and Therapy is an international, peer-reviewed open access journal focusing on breast cancer research, identification of therapeutic targets and the optimal use of preventative and integrated treatment interventions to achieve improved outcomes, enhanced survival and quality of life for the cancer patient.

The manuscript management system is completely online and includes a very quick and fair peer-review system, which is all easy to use. Visit $\mathrm{http}: / /$ www.dovepress.com/testimonials.php to read real quotes from published authors. 